\title{
HIGH RESOLUTION STUDIES OF TYPE III SOLAR RADIO BURSTS
}

\author{
C. CHIUDERI and R. GIACHETTI \\ Osservatorio Astrofisico di Arcetri, Firenze, Italy \\ C. MERCIER \\ Observatoire de Paris, Meudon, France \\ and \\ H. ROSENBERG* and C. SLOTTJE \\ Sterrewacht 'Sonnenborgh', Utrecht, The Netherlands
}

\begin{abstract}
Solar Phys.). High spectral, temporal and spatial resolution observations were obtained with the 60-channel Utrecht solar radio spectrograph (160-320 MHz) and the $169 \mathrm{MHz}$ Nançay solar radioheliograph. From a large number of type III bursts the average height was found to be 0.37 solar radius above the photosphere, corresponding to approximately the Newkirk streamer density, if the bursts are emitted at the harmonic of the local plasma frequency. No center-to-limb variation, nor east-west asymmetry was observed. All double bursts, double humped bursts, precursor-type III had exactly the same position and general shape for both members of the pair. From this it was concluded that fundamental-harmonic pairs are very rare at frequencies above $160 \mathrm{MHz}$ (Mercier and Rosenberg, 1974).

An analysis of the fine structure in decametric type IIIb-type III pairs indicates that the type IIIb burst is related to the fundamental of the plasma frequency, while the type III burst is emitted at the harmonic of the local plasma frequency. The type IIIb fine structure is explained by emission at the same frequency of the plasma frequency and the lowest harmonics of the electron gyro-frequency, through the coupling of plasma waves and low harmonics electron Bernstein waves. The derived densities and magnetic field strengths satisfy lateral pressure equilibrium across the neutral sheet in a coronal streamer. This is regarded as circumstantial evidence for the occurrence of type III bursts inside neutral sheets in coronal streamers (Rosenberg, 1973).

It is shown that a $100 \mathrm{keV}$ electron beam can generate Bernstein waves under coronal conditions. Only the lowest two or three harmonics are rendered unstable, as is observed in the fine structures of type IIIb bursts (Chiuderi et al., 1974).

Recent spectral observations of type III bursts between 160 and $320 \mathrm{MHz}$ show circular polarization up to $100 \%$ during the rise of the type III burst. The degree of polarization falls off exponentially with a time constant of approximately 0.1 (Slottje, 1974).
\end{abstract}

* Presently at Harvard College Observatory, Cambridge, Mass., U.S.A.

Gordon Newkirk, Jr. (ed.), Coronal Disturbances, 225-226. All Rights Reserved.

Copyright $\mathrm{C} 1974$ by the IAU. 


\section{References}

Chiuderi, C., Giachetti, R., and Rosenberg, H.: 1974, to be published.

Mercier, C. and Rosenberg, H.: 1974, to be published.

Rosenberg, H.: 1973, Thesis, Utrecht.

Slottje, C.: 1974, Astron. Astrophys. 32, 107.

\section{COMMENTS}

Leblanc: You are referring to electron density models of a streamer given by Newkirk in $1958(2$ times the quiet corona). I wish to state that there is a large scatter between quiet corona models (Leblanc, Leroy, and Lecautet: 1973, in press, Solar Phys.). These models refer to the period 1970-1972, and we show that they differ from Newkirk's model of the last maximum. Moreover, one streamer model differs from the other. You find that the pairs of type III's are in the same positions, and you conclude that the hypothesis of pairs of fundamental and harmonic components is excluded. I feel that your arguments are not strong enough because when taking scattering into account (Riddle and Leblanc), we could find fundamental and harmonic at the same position. Even the size criterion is not sufficient due to uncertainties in the measurements.

Rosenberg: Our model is Newkirk's 1952 streamer model and not a quiet corona model. We find pairs all over the disk. If there were scattering effects there should be a center-limb effect.

Newkirk: To your knowledge, is there any evidence for curvature in the trajectories of type III bursts as might be expected if they propagate up the legs of streamers on their way up to the nearly radial current sheet?

Rosenberg: As yet, no. Only two-dimensional, multi-frequency observations can show this. Perhaps, with the three-frequency Culgoora array we might see this.

Stewart: We have never seen any trace of highly polarized type III's at Culgoora.

Rosenberg: To see them you need a high time resolution. 